\title{
IMPROVED ADAPTIVE MARKOV RANDOM FIELD BASED SUPER-RESOLUTION MAPPING FOR MANGROVE TREE EXTRACTION
}

\author{
H. Aghighi ${ }^{\text {a,c }} *$ J. Trinder ${ }^{\text {a }}$, S. Lim ${ }^{\text {a }}$, Y. Tarabalka ${ }^{\text {b }}$
${ }^{a}$ School of Civil and Environmental Engineering, The University of New South Wales, UNSW SYDNEY NSW 2052, Australia h.aghighi@student.unsw.edu.au,j.trinder@unsw.edu.au, s.lim@unsw.edu.au
b Inria Sophia-Antipolis Méditerranée, AYIN team, 06902 Sophia Antipolis, France- yuliya.tarabalka@inria.fr
${ }^{c}$ Department of Remote Sensing \& GIS, Faculty of Earth science, Shahid Beheshti University, Tehran, Iran

\section{Commission VIII, WG VIII/8}

KEY WORDS: Super-resolution mapping, Markov random field, Mangrove forest

\begin{abstract}
:
Traditionally, forest tree crowns are extracted using airborne or spaceborne hyper-/multi-spectral remotely sensed images or pansharpened images. However, these medium/low spatial resolution images suffer from the mixed pixel problem, and the cost to collect very high resolution image collection is high. Moreover, existing feature extraction techniques cannot extract local patterns from medium/low resolution images. Therefore, super-resolution mapping (SRM) techniques, which generate land-cover maps with finer spatial resolution than the original remotely sensed image, can be beneficial for the extraction of forest trees. The SRM methods can improve the quality of information extraction by combining spectral information and spatial context into image classification problems. In this paper we have improved an adaptive Markov random field approach for super-resolution mapping (MRF-SRM) based on spatially adaptive MRF-SPM to overcome the limitation of equal covariance matrices assumption for all classes. We applied the developed method for mangrove tree identification from multispectral image recorded by QuickBird satellite, where we generated a super-resolution map with the panchromatic image spatial resolution of $0.6 \mathrm{~m}$. Moreover, the performance of the proposed technique is evaluated by employing the simulated image with different covariance matrices for each class. Our experimental results have demonstrated that the new adaptive MRF-SRM method has increased the overall accuracy by $5.1 \%$ and the termination conditions of this method were satisfied three times faster when compared to the state-of-the-art methods.
\end{abstract}

\section{INTRODUCTION}

Deforestation or removal of forests or trees stands has adverse impacts on the water cycle, ecosystem services, biodiversity, and global biogeochemical cycles, leading to droughts, increased carbon emissions, with consequent impact on climate change [Bagley et al., 2014, Jepma C.J., 2014, Mas et al., 2004]. For instance, deforestation for agricultural purposes in the Brazilian Amazon was an important source of increased annual carbon flux over the period 1989-1998 [Houghton et al., 2000]. Thus, deforestation and forest degradation in the Brazilian Amazon have been observed using remotely sensed data.

Existing deforestation maps in Brazil were generated from medium or low resolution satellite images and do not contains local scale information [Li et al., 2013]. For example, Brazilian Space Agency (INPE) produced the deforestation map for the period of 1988 and 1998 by visual interpretation using Landsat thematic mapper data as well as classification of Landsat multi-spectral scanner (MSS) in 1986 [Houghton et al., 2000]. Another limitation with the use of medium and coarse pixel resolution images is the mixed pixels problem [Li et al., 2013], which do not enable the application of normal feature extraction techniques to utilize the hyper- and multi-spectral data for extraction of local scale information of features as well as individual tree crowns (ITC). This information plays a dominant role in maintaining biotic diversity data [Clark et al., 2005], forest management, species identification, gap analysis, and volume and biomass estimations [Jing et al., 2012]. Another example of the limitation of medium spatial resolution images was demonstrated for many parts of Africa, where the ITC were not observable within a $30 \mathrm{~m}$ resolution

${ }^{*}$ Corresponding author.
Landsat TM images [Houghton, 2005]. In order to solve the mixed pixels problem, soft classification methods can be applied. However, they cannot determine the spatial patterns of a forest within each coarse pixel [Li et al., 2013] and it is not possible to extract the ITC.

The ITC delineation from remote sensing data requires high spatial resolution overlapping photographs/imagery derived from aerial cameras [Halounov, 2003], videography, multispectral airborne or spaceborne sensors [Clark et al., 2005] and elevation data derived from laser scanning or by GNSS field measurement [Ardila et al., 2011]. Although the extracted ITC from high resolution data presents more information than medium resolution image, it does not solve the problem caused by low/ medium resolution multispectral imagery. Moreover, acquiring high resolution time series data sets for a large area is costly and may be impossible [Li et al., 2013].

To date various methods have been introduced for ITC delineation, such as image fusion and multisource multi-resolution classification methods [Lu and Weng, 2007, Solberg et al., 1996, Lu and Weng, 2005, Zhang, 2010], between-tree shadow identification [Warner et al., 1998], region grouping [Erikson, 2003], edge detection [Koch et al., 2006], watershed segmentation [Jing et al., 2012], 3D modelling [Gong et al., 2002], and multi-scale segmentation [Jing et al., 2012]. However, they have not produced a fine resolution ITC map from a coarse resolution image. It should be noted that the pan-sharpening methods which have been used to enhance the spatial resolution of multi-spectral images, can change the spectral information and very often generate artefacts in the images [Tolpekin et al., 2010]. Moreover, these methods need both high resolution panchromatic and multispectral data. Thus, ITC delineation and forest spatial patterns extrac- 
tion within coarse pixels are open challenges.

In this paper, we employed super-resolution mapping (SRM) [Tatem et al., 2002], also called sub-pixel mapping [Verhoeye and De Wulf, 2002] which is a land cover classification technique to produce the classified map at a finer spatial resolution than the original coarse resolution image. The idea of SRM was introduced by Atkinson [Atkinson, 1997] to achieve sub-pixel vector boundaries using spatial dependence maximization. In general, spatial dependence means that the neighboring pixels belong, with the high probability, to the same class as observations further apart [Atkinson, 1991]. Later on, several attempts have been made to utilize this theory and employ a method to tackle the SRM issue. Generally, SRM methods can be divided into two main categories [Li et al., 2012]: 1) methods which are applied as post-processing algorithms and require soft classification results, and 2) those which can be categorised as a classification and are independent of soft classification methods.

The first approach can be divided into methods based on sub-pixel swapping [Luciani and Chen, 2011, Shen et al., 2009, Thornton et al., 2006, Yong and Bo, 2014]; multiple endmember spectral mixture analysis [Powell et al., 2007]; geostatistics [Atkinson et al., 2008, Boucher and Kyriakidis, 2006, Boucher et al., 2008, Qunming et al., 2014]; the broad category of spatial attraction [Liguo et al., 2011, Mertens et al., 2006, Mertens et al., 2003a,Ling et al., 2013, Wang et al., 2012]; and those based on utilizing heuristic methods to improve the $S R M$ accuracy by maximizing the spatial dependence and generating the spatial distribution of land cover within the mixed pixels [Mertens et al., 2003b, Tatem et al., 2001, Zhang et al., 2008, Zhong and Zhang, 2013]. In contrast, the second SRM approaches do not rely on the availability of accurate class boundaries nor a sub-pixel classified map derived by another method [Kasetkasem et al., 2005].

In this paper, we consider the second category for defining contextual $S R M$, which was proposed by [Kasetkasem et al., 2005], and employs Markov random field $(M R F)$ because of its suitability to represent the spatial dependence between pixels. This method was developed based on three main assumptions: the pixels of the fine spatial resolution image are pure, SRM satisfies the $M R F$ properties, and the pixel intensities for each class in the fine resolution image are normally distributed. In contrast to the first SRM type, referred to above the results of this method does not rely on the availability of accurate class boundaries nor a subpixel classified map derived by another method [Kasetkasem et al., 2005].

In this paper we adopt $S R M$ described in [Tolpekin and Stein, 2009] and the spatially adaptive $M R F-S P M$ proposed in [Li et al., 2012] both of which have proved to work well on simulated Gaussian distributed multispectral images. These methods were developed based on equal covariance matrix between the classes. The first novelty of this work consists in employing the proposed method for the ITC mapping in the mangrove forests. The second novelty of this work is that it utilizes multispectral QuickBird images with a resolution of $2.4 \mathrm{~m}$, instead of the synthetic image used by [Tolpekin and Stein, 2009, Li et al., 2012], to produce the mangrove forest map at the panchromatic imagery spatial resolution of $0.6 \mathrm{~m}$. Finally, we have attempted to overcome the limitation of the equal covariance matrix assumption between classes used by [Tolpekin and Stein, 2009, Li et al., 2012].

The outline of this paper is as follows: Section 2. introduces the basic of $M R F-S R M$ frame work and explicitly explains the frame work of improved adaptive $M R F-S R M$. The data description and the experimental results are presented and discussed in Section 3. Finally, conclusions are drawn in Section 4.

\section{PROPOSED METHOD}

In the development of MRF based SRM framework, we denote an image by $Y=\left\{\mathscr{Y}_{i} \in R^{B}, i=1,2, \ldots, m\right\}$, where $B$ is a number of spectral channels, and $m=N_{1} \times N_{2}$ is a number of pixels in an image. The spatial resolution of image $Y$ is denoted as $R$; therefore each pixel $\mathscr{Y}_{i}$ represents a square area of size $R^{2}$ on the ground. It is assumed that the spectral intensity of each pixel $\mathscr{Y}_{i}$ depends on a corresponding unobserved pixel label in $L=$ $\left\{\ell_{j}, j=1,2, \ldots, m\right\}$ [Bouman and Shapiro, 1994], where, each $\ell_{j}$ takes its value from a finite set of $M$ thematic classes of interest $\Omega=\left\{\omega_{1}, \omega_{2}, \ldots, \omega_{M}\right\}$. Although image $Y$ was captured by an airborne or spaceborne sensor, we assume that this image was generated by degradation of a not directly observed image $(X)$ with the same number of spectral bands and spatial resolution $r$. We assume that every pixel of $X$ is pure and can be assigned to a unique class [Tolpekin and Stein, 2009]. It is also assumed that the spectral intensities of the pixels of $X$ and $Y$ which belong to the same class, are spatially uncorrelated [Tolpekin and Stein, 2009].

The ratio between the coarse pixel spatial resolution $(R)$ and fine pixel spatial resolution $(r)$ image is called the scale factor $(S=$ $R / r)$ and is assumed to be an integer value [Tolpekin and Stein, 2009]. Hence, each coarse pixel of Y consists of $S^{2}$ pixels of $X$ and the corresponding positions of fine pixels within $\mathscr{Y}_{i}$ can be indexed by $x_{k \mid i}$, where $k=1,2, \ldots, S^{2}$. By excluding the partial overlaps between the coarse and fine pixels, the relationship between each coarse pixel of $Y$ and its corresponding finer pixels of $X$ can be established as follows [Li et al., 2012]:

$$
\mathscr{Y}_{i}=\frac{1}{S^{2}} \sum_{k=1}^{S^{2}} x_{k \mid i}
$$

\subsection{MRF-SRM}

The aim of $S R M$ is to produce a classified map $C_{S R M}$ at a finer spatial resolution $(r)$, the same as $X$, from a coarse resolution image $(Y)$ [Tolpekin and Stein, 2009]. For this reason we employed the Bayes' rule:

$$
p\left(C_{S R M} \mid Y\right) \propto p\left(Y \mid C_{S R M}\right) p\left(C_{S R M}\right)
$$

where $p\left(C_{S R M} \mid Y\right)$ is the posterior probability of the classified $S R$ map, $p\left(Y \mid C_{S R M}\right)$ is the class-conditional probability and $p\left(C_{S R M}\right)$ is the prior probability distribution for the $S R$ map $C_{S R M}$. The optimal classified $S R$ map $C_{S R M}^{*}$ given the image $Y$ can be generated by solving the maximization problem for the a posteriori probability $(M A P)$ decision rule (2):

$$
C_{S R M}^{*}=\underset{C_{S R M}}{\operatorname{argmax}}\left\{p\left(C_{S R M} \mid Y\right)\right\}=\underset{C_{S R M}}{\operatorname{argmax}}\left\{p\left(Y \mid C_{S R M}\right) p\left(C_{S R M}\right)\right\}
$$

According to the complexity of (3) which involves the optimization of a global distribution of the image and due to the equivalence of $M R F$ and Gibbs random field, this optimization can be resolved by minimizing the sum of local posterior energies, as proposed in [Tolpekin and Stein, 2009]:

$$
U\left(C_{S R M} \mid Y\right)=U\left(Y \mid C_{S R M}\right)+U\left(C_{S R M}\right)
$$

where $U\left(C_{S R M} \mid Y\right)$ is the posterior energy function, $p\left(U \mid C_{S R M}\right)$ is the spectral energy function, and $U\left(C_{S R M}\right)$ is the spatial energy function (prior energy function). The spatial term $U\left(C_{S R M}\right)$ is defined by using the Potts model, which penalizes different class 
labels for neighboring sub-pixels:

$$
\begin{array}{r}
U\left(C_{S R M}\right)=\sum_{k, i} U\left(C_{S R M}\left(a_{k \mid i}\right)\right)= \\
\sum_{k, i} \sum_{a_{l} \in N\left(a_{k \mid i}\right)} q \times \phi\left(a_{l}\right) \times\left(1-\delta\left(C_{S R M}\left(a_{k \mid i}\right), C_{S R M}\left(a_{l}\right)\right)\right)
\end{array}
$$

Here, $U\left(C_{S R M}\left(a_{k \mid i}\right)\right)$ is the local spatial energy of the sub-pixel $a_{k \mid i}$ and $C_{S R M}\left(a_{k \mid i}\right)$ is the class label of sub-pixel $a_{k \mid i}$ within a coarse pixel $\mathscr{Y}_{i}$, and $C_{S R M}\left(a_{l}\right)$ is the class label of its surrounding neighbors. In this equation, $\delta\left(\ell_{i}, \ell_{j}\right)$ is the Kronecker delta function, $\left(\delta\left(\ell_{i}, \ell_{j}\right)=1\right.$ if $\ell_{\mathrm{i}}=\ell_{\mathrm{j}}$ and $\delta\left(\ell_{i}, \ell_{j}\right)=0$ if $\left.\ell_{\mathrm{i}} \neq \ell_{\mathrm{j}}\right), q$ controls the overall magnitude of weights and consequently the contribution of the spatial energies $0 \leq q<\infty$, and $\phi\left(a_{l}\right)$ denotes the weight of contribution from sub-pixel $a_{l} \in N\left(a_{k \mid i}\right)$ to the spatial energy term and can be computed as (6):

$$
\phi\left(a_{l}\right)=\frac{1}{\eta}\left(\frac{d\left(a_{k \mid i}, a_{l}\right)}{r}\right)^{-g}
$$

where $d\left(a_{k \mid i}, a_{l}\right)$ denotes the geometric distance between the sub-pixel $a_{k \mid i}$ and its spatial neighbors $a_{l}$ [Li et al., 2012, Makido et al., 2007], $\eta$ is the normalization constant so that $\sum_{l \in N\left(a_{k \mid i}\right)} \eta\left(a_{l}\right)$ $=1$, and the power law index $g$ is usually set as $g=1$ [Li et al., 2012]. The spectral energy term $U\left(Y \mid C_{S R M}\right)$ can be expressed as:

$$
\begin{aligned}
& U_{\text {Spectral }}\left(Y \mid C_{S R M}\right)=\sum_{i, k} U\left(\mathscr{Y}_{i} \mid C_{S R M}\left(a_{k \mid i}\right)\right) \\
& =\sum_{i, k}\left[\frac{1}{2}\left(\mathscr{Y}_{i}-\mu_{i}\right)^{\prime} \Sigma_{i}^{-1}\left(\mathscr{Y}_{i}-\mu_{i}\right)+\frac{1}{2} \ln \left|\Sigma_{i}\right|\right]
\end{aligned}
$$

where $\mathscr{Y}_{i}$ is the spectral vector of the coarse pixel $i$, assumed to be normally distributed with mean $\mu_{i}$ and covariance $\Sigma_{i}$. Both $\mu_{i}$ and $\Sigma_{i}$ are dependent on the pixel composition and can be computed using [Tolpekin and Stein, 2009]:

$$
\begin{aligned}
& \mu_{i}=\sum_{\alpha=1}^{M} \theta_{\alpha i} \mu_{\alpha} \\
& \Sigma_{i}=\sum_{\alpha=1}^{M} \theta_{\alpha i} \Sigma_{\alpha}
\end{aligned}
$$

where $\theta_{\alpha i}$ is the proportion of the class $\omega_{\alpha}$ in the composition of coarse pixel $\mathscr{Y}_{i}$, such that $\sum_{\alpha=1}^{M} \theta_{\alpha i}=1$, and $\mu_{\alpha}$ and $\Sigma_{\alpha}$ are the mean and covariance of the class $\omega_{\alpha}$, which are estimated using the sufficient number of pure training pixels [Kasetkasem et al., 2005]. By substituting (5, 7) in (4), we can write (4) as:

$$
\begin{array}{r}
U\left(C_{S R M} \mid Y\right)=U\left(Y \mid C_{S R M}\right)+q \sum_{k, i} \sum_{a_{l} \in N\left(a_{k \mid i}\right)} \phi\left(a_{l}\right) \times \\
\left(1-\delta\left(C_{S R M}\left(a_{k \mid i}\right), C_{S R M}\left(a_{l}\right)\right)\right)
\end{array}
$$

In order to normalize $U\left(C_{S R M} \mid Y\right)$, we multiply (10) by $1 /(1+q)$ :

$$
\begin{array}{r}
U\left(C_{S R M} \mid Y\right) \propto \frac{1}{1+q} U\left(Y \mid C_{S R M}\right)+\frac{q}{1+q} \sum_{k, i} \sum_{a_{l} \in N\left(a_{k \mid i}\right)} \phi\left(a_{l}\right) \times \\
\left(1-\delta\left(C_{S R M}\left(a_{k \mid i}\right), C_{S R M}\left(a_{l}\right)\right)\right)
\end{array}
$$

In this step we call $q /(1+q)$ smoothing parameter which is denoted as $\lambda$; hence $1 /(1+q)$ can be expressed as $1-\lambda$. As mentioned in Equation (3), the optimal SR map $C_{S R M}^{*}$ depends on the maximizing the posterior probability which is similar to minimization of posterior energy $U\left(C_{S R M} \mid Y\right)$, not the absolute value of $U\left(C_{S R M} \mid Y\right)$ [Tolpekin and Stein, 2009]; thus, equation (11) can be written as :

$$
\begin{array}{r}
U\left(C_{S R M} \mid Y\right)=(1-\lambda) U\left(Y \mid C_{S R M}\right)+\lambda \sum_{k, i} \sum_{a_{l} \in N\left(a_{k \mid i}\right)} \phi\left(a_{l}\right) \times \\
\left(1-\delta\left(C_{S R M}\left(a_{k \mid i}\right), C_{S R M}\left(a_{l}\right)\right)\right)
\end{array}
$$

\subsection{Adaptive MRF-SRM}

According to Equations (5) and (7) the spatial and spectral energies which are required to compute posterior energy in Equation (12) should be computed for each coarse pixel. However, $\lambda$ in (12) was a fixed value for the entire image by [Tolpekin and Stein, 2009]. Thus, by estimating a smoothing parameter for each coarse pixel using its local contextual information, we can modify the MRF-SRM model (12) into an adaptive MRF-SRM [Li et al., 2012]. For this reason, assume that the class label of a given sub-pixel $C_{S R M}\left(a_{k \mid i}\right)=\alpha$ is assigned to an incorrect class label $C_{S R M}\left(a_{k \mid i}\right)=\beta$ within a coarse pixel $\mathscr{Y}_{i}$. Therefore, based on (3) we can infer that:

$$
U\left(C_{S R M}\left(a_{k \mid i}\right)=\alpha \mid \mathscr{Y}_{i}\right) \geq U\left(C_{S R M}\left(a_{k \mid i}\right)=\beta \mid \mathscr{Y}_{i}\right)
$$

If this condition is not corrected, then an incorrect class label will be assigned by the MAP solution to $a_{k \mid i}$. Substituting the corresponding terms in (13) and solving this inequality equation, we will have changes in the spectral energy $\Delta U_{\alpha \beta}^{\text {spec }}$ and in the spatial energy $\Delta U_{\alpha \beta}^{s p a t}$ [Tolpekin and Stein, 2009]; where, $\Delta U_{\alpha \beta}^{\text {spec }}$ can be computed using (14) and $\Delta U_{\alpha \beta}^{\text {spat }}$ using (15) [Tolpekin and Stein, 2009, Li et al., 2012].

$$
\Delta U_{\alpha \beta}^{\text {spec }}=\frac{1}{2}\left(\frac{\mu_{\beta}-\mu_{\alpha}}{S^{2}}\right)^{\prime} \Sigma_{i}^{-1}\left(\frac{\mu_{\beta}-\mu_{\alpha}}{S^{2}}\right)
$$

where due to equal covariance matrix assumption $\Sigma_{i}=\left(1 / S^{2}\right) \Sigma_{\alpha}$ [Tolpekin and Stein, 2009] Moreover, they proposed Equation (15) to compute the change in spatial energy $\Delta U_{\alpha \beta}^{\text {spat }}$ from class $\alpha$ to $\beta$ [Tolpekin and Stein, 2009] .

$\Delta U_{\alpha \beta}^{s p a t}=q\left|\sum_{a_{l} \in N\left(a_{k \mid i}\right)} \phi\left(a_{l}\right)\left[\delta\left(\beta, C_{S R M}\left(a_{l}\right)\right)-\delta\left(\alpha, C_{S R M}\left(a_{l}\right)\right)\right]\right|$

where parameter $\gamma$ is related to the prior energy coefficient $\phi$, the neighbouring window size $(W=2 S-1)$ and the configuration of pixel class label $C_{S R M}\left(a_{l}\right)$ in the $N\left(a_{k \mid i}\right)$ of a specific image [Tolpekin and Stein, 2009]. Furthermore, $\gamma$ for each pair of classes $(\alpha$ and $\beta$ ) can be estimated as [Tolpekin and Stein, 2009]:

$$
\lambda^{*}=\frac{1}{1+\frac{\gamma}{\Delta \mathrm{U}_{\alpha \beta}^{\mathrm{spec}}}}
$$

Because $\lambda^{*}$ is a fixed value for the entire image, [Li et al., 2012] employed the proportions of land cover classes within each coarse 
pixel to compute the mean of spectral energy change $\Delta U_{i}^{S p e c}$ within each coarse pixel:

$$
\Delta U_{i}^{S p e c}=\frac{\sum_{\alpha=1}^{M-1} \sum_{\beta=\alpha+1}^{M} \theta_{\alpha i} \theta_{\beta i} \Delta U_{\alpha \beta}^{\text {Spec }}}{\sum_{\alpha=1}^{M-1} \sum_{\beta=\alpha+1}^{M} \theta_{\alpha i} \theta_{\beta i}}
$$

Then, they modified (16) as:

$$
\lambda_{i}^{*}=\frac{1}{1+\frac{\gamma}{\Delta U_{i}^{S p e c}}}
$$

Equation (18) computes the smoothing parameter for each coarse pixel $\mathscr{Y}_{i}$. Hence, this method can be considered as an adaptive super resolution mapping method. However, it should be noted that the spectra statistics of classes show that the mean and covariance of classes are different; thus, the maximum likelihood estimation utilizes the likelihood energy (7) to assign a class to a given pixel based on minimum distance [Richards and Jia, 2006]. However, both methods [Tolpekin and Stein, 2009,Li et al., 2012] suffer from the assumption of the same class covariance matrix in Equation (14); thus, they employed the Mahalanobis distance (14) to estimate the spectral energy change from a correct class to an incorrect class label $\Delta U_{\alpha \beta}^{s p e c}$. To overcome this limitation, we propose $\Sigma_{\alpha \beta}$ which is the average of covariance matrix of classes $\alpha$ and $\beta$, instead of using the same covariance matrix for all the classes:

$$
\Sigma_{\alpha \beta}=\frac{\Sigma_{\alpha}+\Sigma_{\beta}}{2}
$$

By employing (19), Equation (14) can be rewritten as (20).

$$
\Delta U_{\alpha \beta}^{\text {spec }}=\frac{1}{2}\left(\frac{\mu_{\beta}-\mu_{\alpha}}{S^{2}}\right)^{\prime} \Sigma_{\alpha \beta}{ }^{-1}\left(\frac{\mu_{\beta}-\mu_{\alpha}}{S^{2}}\right)
$$

By utilizing (20) instead of (14), our adaptive MRF-SRM is not dependent on the same covariance matrix which assumed by [Tolpekin and Stein, 2009, Li et al., 2012]. Moreover, it can be applied on a real image with a different covariance matrix for each class to produce a $S R$ map.

\subsection{Optimization and Estimation}

In this work, we employed simulated annealing (SA) as a heuristic optimization technique to iteratively search for a new solution (see Figure 1). The simulated annealing schedule is based on the following power-law decay function:

$$
T_{\text {iter }}=\sigma T_{\text {iter-1 }},
$$

where $T_{\text {iter }}$ is the temperature at the iteration number iter, and $\sigma \in(0,1)$ controls the rate of temperature decrease. In the first step of this method, an initial $S R$ map should be produced. For this reason, we employed a constraint linear spectral unmixing (LSU) method to generate the initial class proportion of each coarse pixel (Figure $3(\mathrm{~d})$ ). Then the scale factor and estimated fractional abundance $\left(F A_{j}\right)$ results for each coarse pixel were utilized to estimate the number of sub-pixels $N S P_{j}$ for each class $j$ within a coarse pixel $\mathscr{Y}_{i}$ :

$$
N S P_{j}=\operatorname{round}\left(\frac{F A_{j}}{1 / S}\right)
$$

where round $(\bullet)$ returns the value of the closest integer. Then, we randomly assign a number $N S P_{j}$ of sub-pixels within a coarse pixel $\mathscr{Y}_{i}$ : to class $\ell_{j}$. We called the generated map as the initial $S R$ map $C_{S R M}^{\text {Init }}\left(a_{k \mid i}\right)$. In the next step, we compute the spectral energy change $\Delta U_{\alpha \beta}^{s p e c}$ using (20) and the mean of spectral change $\Delta U_{i}^{\text {spec }}$ using (17) to estimate the optimal smoothing parameter $\lambda_{i}^{*}$ for each coarse pixel by (18). Then, $\lambda_{i}^{*}$ was used to compute the posterior energy (12) of each coarse pixel. Before starting the iterative steps, some parameters should be defined: the starting iteration number $\left(\right.$ iter $_{\text {start }}=1$ ), maximum number of iterations $\left(\right.$ iter $_{\max }$ ), starting temperature $T_{0}=2$, the temperature $\sigma=0.9$. The SA is performed until it satisfies the termination conditions: 1) iter $\geq$ iter $_{\text {max }}$; 2) less than $0.1 \%$ of the sub-pixels are successfully updated during three consecutive iterations.

In order to utilize the SA algorithm, a change in a sub-pixel class label $C_{S R M}\left(a_{k \mid i}\right)$ is performed to produce a new solution. Consequently, a new posterior energy (12) is computed. The change will be accepted if it decreases the energy, and it will be accepted with a certain probability if it increases the posterior energy. The iterative procedure continues until the model satisfies one of the convergences conditions.

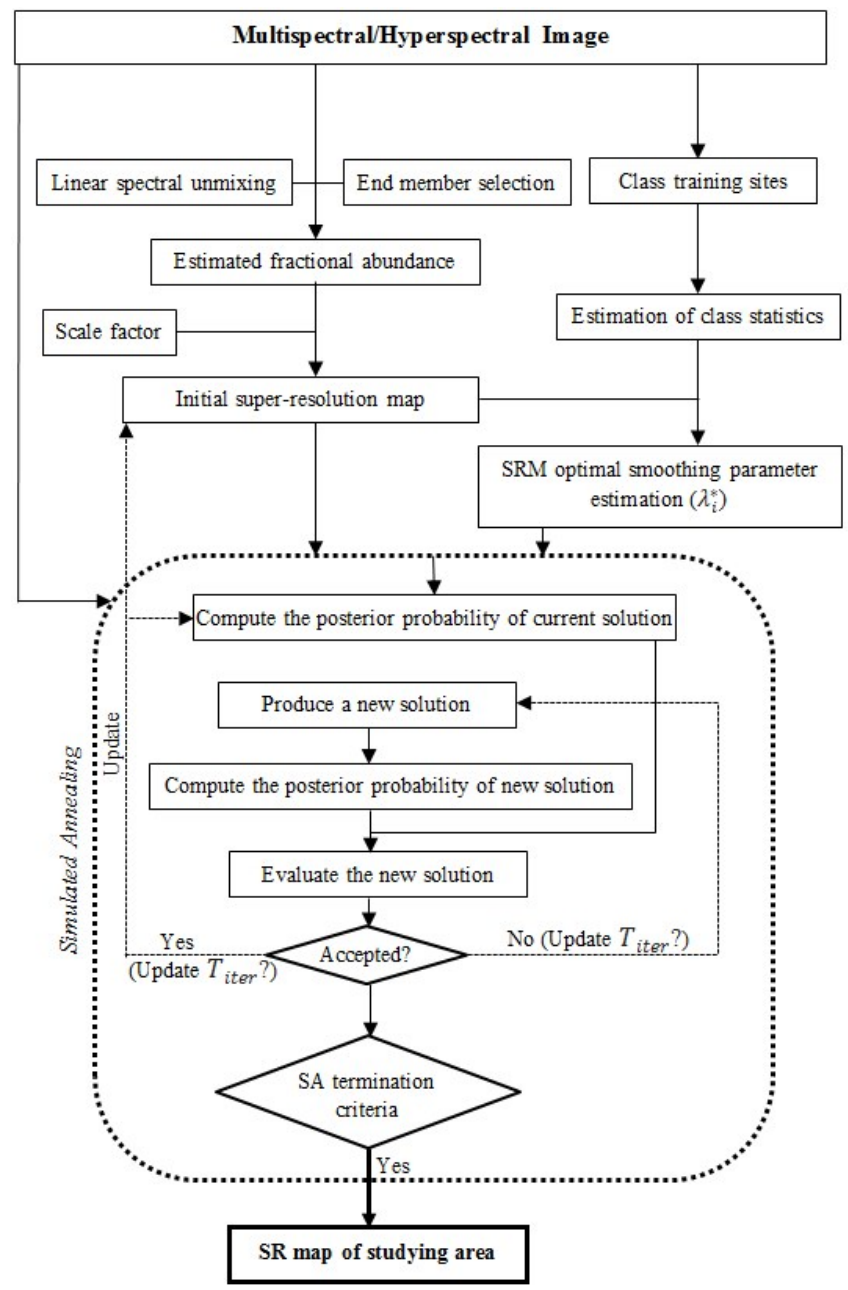

Figure 1: Workflow of adaptive super-resolution mapping

\section{EXPERIMENTAL RESULTS AND DISCUSSION}

In order to evaluate the performance of the proposed method, two different datasets were selected.

1) Mangrove forest dataset: This image was recorded by the QuickBird satellite over the mangrove forest in the south east 
of Iran, which in UTM projection is located between, ULX: 347949.3, ULY: 2786168.1 and LRX: 348126.9, LRY: 2785990.5 on April 06, 2006. The studying area is located within Govatr Bay in the delta of Bahookalat River which is a part of Gandoo Protected Area and Bahoo wetland and demonstrated as position 10 in Figure 2 [Zahed et al., 2010]. The satellite simultaneously captures panchromatic (Pan) and multispectral (MS) digital imagery with spatial resolution of 0.6 and $2.4 \mathrm{~m}$ at nadir, respectively. Thus, the scale factor $S$ between MS (Figure 3(a)) and Pan (Figure 3(b)) images is 4 . The Pan sensor captures the surface reflectance within a wavelength range of 450 to $900 \mathrm{~nm}$, while the MS sensor provides four spectral bands; i.e. blue $(450-520 \mathrm{~nm})$, green $(520-600 \mathrm{~nm})$, red $(630-690 \mathrm{~nm})$, near-IR $(760-890 \mathrm{~nm})$. The selected area comprises 300 by 300 panchromatic pixels and their corresponding multispectral pixels. In this dataset, we tried to select a pure studying area with some individual mangrove tree crowns, in the non-dense mangrove forest. Therefore, we were able to extract the trees accurately and evaluate the results. The reference map (Figure 3(c)) was produced using visual interpretation of the panchromatic image and pan sharpening product.

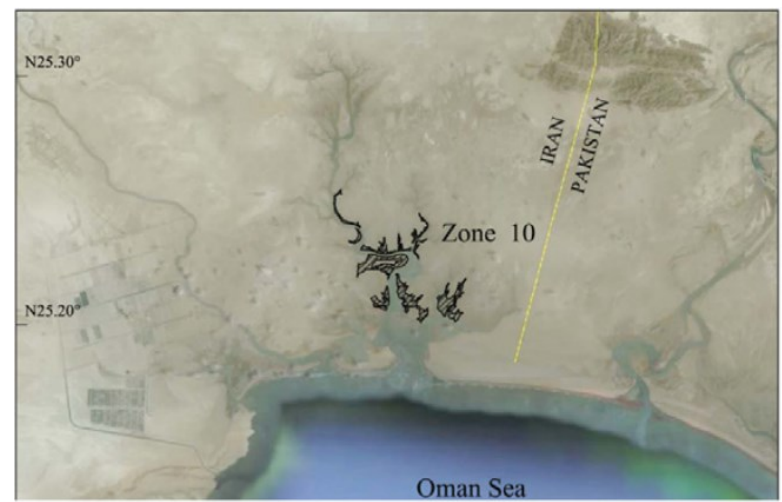

Figure 2: Govatr Bay mangorves forest; adopted from (Zahed et al., 2010)

2) Simulated image: This dataset is a simulated image which was used to compare the accuracy of our improved adaptive $M R F-S R M$ with that of [Li et al., 2013]. In order to generate this dataset, we employed the reference map of mangrove forest dataset comprising 300 by 300 pixels, which contains real tree boundaries. Then, the trees are categorized into two different classes (class 1 and class 2 ) to generate a reference map with complex class conditions (Figure 5(a)). The third class of this map is considered as soil. Then, we employed the (Mohn et al., 1987; Yu and Ekstrm, 2003) methodology as well as the proposed class mean values and class covariance matrix. The location of each distribution is presented as an ellipsoid in Figure 4. Then by utilizing the mean and covariance of the classes, a synthetic image with two bands and three classes was generated by sampling from the multivariate normal distribution. The generated image is degraded by scale factor 6 (Figure 5(b \& c)) and utilized to apply adaptive $M R F-S P M$ and our improved adaptive MRF-SRM.

To estimate the efficiency of the proposed spatially adaptive $M R F$ $S P M$ [Li et al., 2012] and our improved adaptive MRF-SRM method, we applied both methods on the both datasets. Then the overall $(O A)$, average $(A A)$ and class-specific accuracies, as well as the kappa coefficient $(k)$ are estimated. The resulting generated $S R$ maps for mangrove forest dataset are presented in Figure 3(d) and for the simulated image in Figures 5(c) and 5(d). Mangrove forest dataset contains two classes (mangrove and soils), thus the

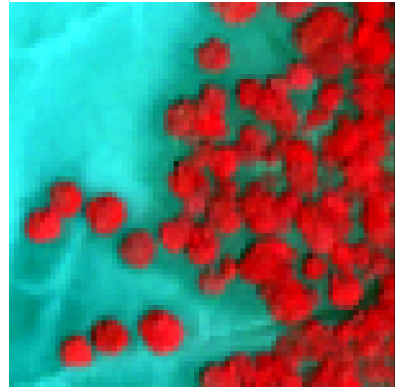

(a)

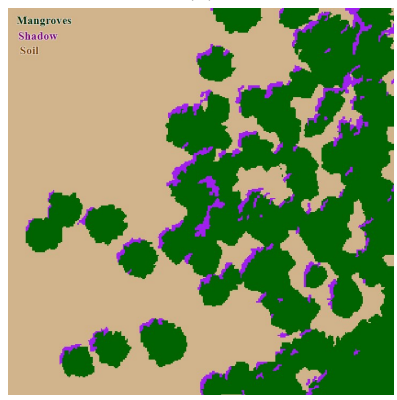

(c)

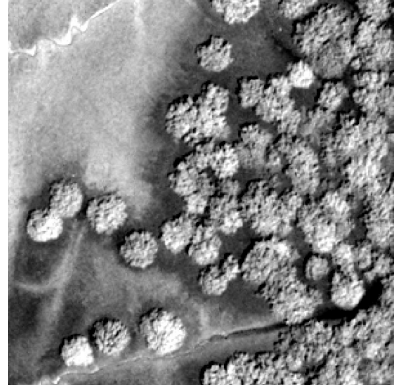

(b)

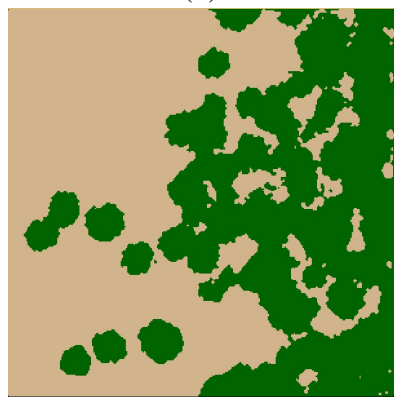

(d)
Figure 3: (a) Three-band colour composite of QuickBird Image. (b) The panchromatic image of QuickBird Image. (c) Reference data of the mangrove forest dataset. (d) Generated $S R$ map using spatially adaptive $M R F-S P M$ method.

results of both spatially adaptive $M R F-S P M$ and our improved adaptive $M R F-S R M$ are the same.

In order to evaluate the performance of both SRM methods, we applied five state-of-the-art classification techniques, namely maximum likelihood $(M L)$, Mahalanobis distance $(M a D)$, minimum distance $(M i D)$, spectral angle mapper $(S A M)$, and spectral correlation mapper $(S C M)$ on the original simulated image and the fused image of MS and Pan for the mangrove forest dataset. All the classification methods were trained with similar training data sets (36 pixels per class) and their performance was evaluated by their corresponding reference maps. The results are reported in Table I, from which, it can be concluded that the improved adaptive $M R F-S R M$ compared to the spatially adaptive $M R F$ $S P M$ increased the overall accuracy by 5.1 percent. Although all the pixel based classification methods were applied on the original simulated image with scale factor 1 instead of scale factor 6 which was utilized for both spatially adaptive $M R F-S P M$ and improved adaptive $M R F-S R M$ methods, the $O A, A A$, and $k$ of both $S R$ generated maps are higher than $S A M, S C M$ and $M i D$ classified maps results (Table 1).

We evaluated the statistical significance of the difference between all the classification results in terms of accuracy by using the $M c$ Nemars test with the 5\% significance level for each pair of the classification maps [Aghighi et al., 2014]. According to the calculated $\chi^{2}$ and $z$ values, the null hypothesis $\left(H_{0}\right)$ of no significant difference between map accuracies is rejected. Thus, the results of improved adaptive $M R F-S R M$ and spatially adaptive $M R F-S P M$ are not the same as each other nor the other classified maps. This means that the use of the proposed improved adaptive $M R F-S R M$ is beneficial to overcome the mixed pixel problem and it is suitable to produce a fine resolution ITC map or to extract the forest spatial patterns within coarse pixels.

As mentioned in Section 1., the image fusion and multisource multi-resolution classification methods are some of the state- 


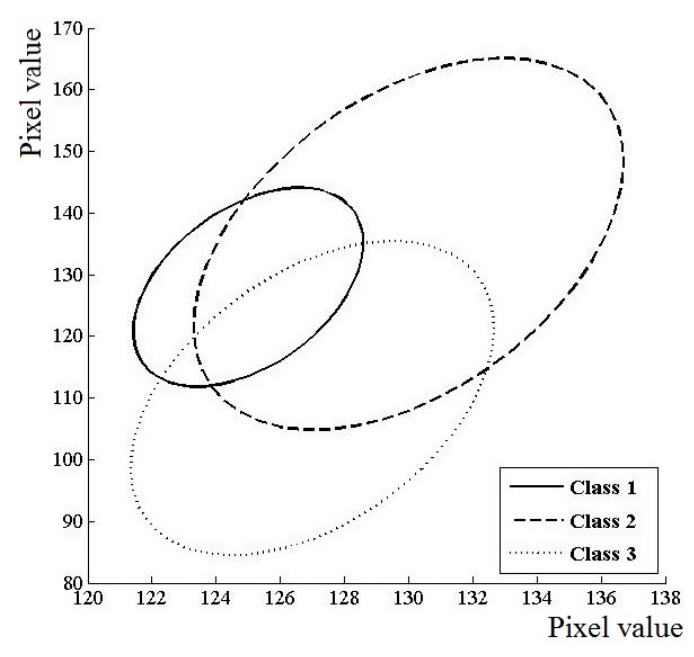

Figure 4: Class expectation \pm 2 standard deviation contours; in the case of spatial independency between pixels.

Table 1: Classification accuracy in percentage for each class of the simulated image, where $O A, A A, k, S A M R F-S P M$, and $I A M R F-S R M$ represent the overall accuracy, average accuracy, kappa coefficient, spatially adaptive $M R F-S P M$, and improved adaptive $M R F-S R M$.

\begin{tabular}{|c|c|c|c|c|c|c|c|c|}
\hline & SA MRF-SPM & IA MRF-SRM & ML & SAM & SCM & MiD & MaD \\
\hline OA & 89 & 94.1 & 96 & 78 & 78.5 & 85.4 & 95.5 \\
AA & 87.4 & 87.8 & 93.6 & 67.5 & 46.6 & 81.5 & 92.6 \\
$K$ & 0.81 & 0.83 & 0.93 & 0.62 & 0.59 & 0.75 & 0.93 \\
\hline C & 1 & 88.3 & 89.7 & 98.8 & 56.4 & 62.4 & 65.9 & 97.4 \\
$C$ & 2 & 83.1 & 84.9 & 93.9 & 46.6 & 37.5 & 79.1 & 81.4 \\
C & 3 & 90.9 & 92.2 & 95.4 & 99.3 & 86.8 & 99.6 & 99.2 \\
\hline
\end{tabular}

of-the-art approaches for ITC delineation. Therefore, we applied different pan-sharpening methods, called subtractive resolution merge, HPF resolution merge, Wavelet resolution merge, and Ehlers resolution merge on mangrove forest dataset pansharpened images. Then, $M L, M a D, M i D, S A M$, and $S C M$ pixel based classification methods were employed to classify the generated pan-sharpened images. In these experiments, the similar training data sets (36 pixels per class) were utilized to train the classification methods and their performance evaluated by the mangrove forest reference maps (Table 2).

From Table 2, it can be seen that the overall accuracy of the generated mangrove forest using the spatially adaptive $M R F-S P M$ methods are higher than the overall accuracy of all classification methods using the pan-sharpened images, except for Subtractive resolution merge employing spectral angle mapper method. The calculated $\chi^{2}$ and $z$ values for spatially adaptive $M R F-S P M$ and each of the other classification methods indicate that the null hypothesis $\left(H_{0}\right)$ of no significant difference between map accuracies are strongly rejected. Thus, both improved adaptive $M R F$ $S R M$ method and spatially adaptive MRF-SPM are suitable to produce a fine resolution mangrove forest map or to extract the forest spatial patterns within coarse pixels. However, due to better performance of the improved adaptive $M R F-S R M$ than spatially adaptive $M R F-S P M$ for the simulated image with non-equal covariance matrices, it can be concluded that the proposed improved adaptive $M R F-S R M$ method could be more suitable than the spatially adaptive $M R F-S P M$ for real images with non-equal covariance matrix for the classes (Table 1). Moreover, the termination conditions of the improved adaptive MRF-SRM were satisfied three times faster when compared to spatially adaptive $M R F-S P M$ for simulated images.

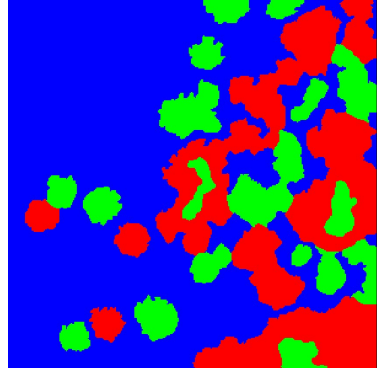

(a)

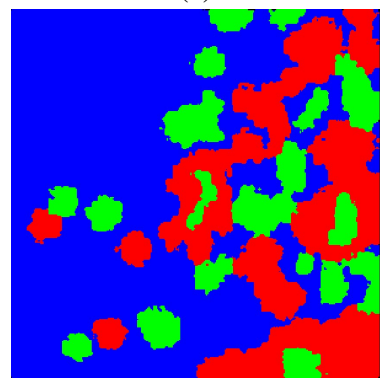

(c)

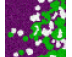

(e)

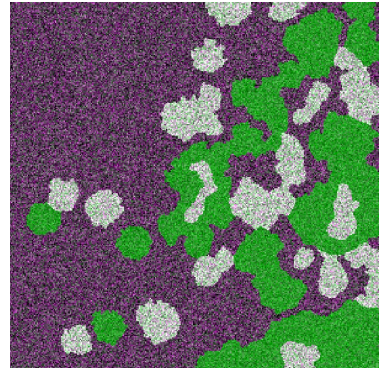

(b)

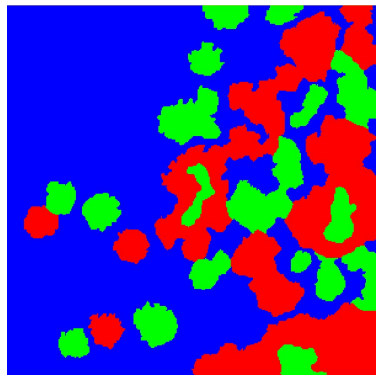

(d)
Figure 5: (a) Reference map of simulated dataset. (b) Simulated image with 2 bands and scale factor 1. (c) Adaptive MRF-SPM result. (d) Improved adaptive MRF-SRM result. (e) The real proportional size of degraded simulated image with scale factor 6 (produced from Figure 5(b)) which is used to generate SR maps presented in Figures $5(\mathrm{c} \& \mathrm{~d})$ ).

\section{CONCLUSION}

In this work, we have investigated the use of the proposed spatially adaptive $M R F-S R M$ to generate finer spatial resolution mangrove forest maps from a multispectral image with lower spatial resolution. This method is applied on QuickBird satellite images over the mangrove forest and its results are compared with 20 different classified maps produced by five different pixel based classification methods on four different pan-sharpened images. Experimental results have demonstrated that the accuracy of the generated mangrove forest maps are mostly better than the results of other techniques.

According to this reason that the covariance matrices of different classes are not same, we modified the earlier method and proposed a new method which is called improved adaptive $M R F$ $S R M$ to overcome this assumption of equal covariance matrices. The performance of this method was evaluated using a two band simulated image with scale factor 6 which contains three different classes with very complex shapes. The generated map using this method is compared with spatially adaptive $M R F-S P M$ using the same dataset and five state-of-the-art pixel based classification methods for the original simulated image with a scale factor 1. Experimental results have demonstrated $O A, A A, k$ of generated $S R$ map by the improved adaptive $M R F-S R M$ method are higher than those of spatially adaptive $M R F-S P M$. Moreover, McNemar's test rejects the null hypothesis $\left(H_{0}\right)$ of no significant difference between them which indicates the statistically significant difference between them at the 0.05 level. 
Table 2: the classification accuracy in percentage for each class of the the mangrove forest image, where $O A, A A, k$, and $S A M R F$ $S P M$ denote the overall accuracy, average accuracy, kappa coefficient, and spatially adaptive $M R F-S P M$.

\begin{tabular}{|c|c|c|c|c|c|c|}
\hline & & & & & & \\
\hline & Subtr & e res & olutio & $\mathrm{n}$ mer & & \\
\hline$O A$ & 92.7 & 87 & 93 & 89.3 & 90.9 & 86.9 \\
\hline$A A$ & 92.1 & 87.2 & 92.5 & 89.1 & 90.3 & 87.2 \\
\hline$K$ & 0.85 & 0.74 & 0.86 & 0.78 & 0.81 & 0.74 \\
\hline 1 & 87.9 & 76.1 & 89.3 & 62.4 & 84.8 & 76 \\
\hline$C 2$ & 96.3 & 98.4 & 95.7 & 37.5 & 95.7 & 98.4 \\
\hline & & solu & ion $\mathrm{m}$ & erge & & \\
\hline$O A$ & 92.7 & 83.3 & 91.7 & 88.8 & 91.9 & 83.3 \\
\hline$A A$ & 92.1 & 85 & 91.1 & 0.78 & 91.3 & 85 \\
\hline$K$ & 0.85 & 0.67 & 0.83 & 88.6 & 0.83 & 0.67 \\
\hline 51 & 87.9 & 70.6 & 87.1 & 79.3 & 87.7 & 70.5 \\
\hline$C 2$ & 96.3 & 99.4 & 95 & 97.8 & 94.9 & 99.5 \\
\hline & Wa & resol & ution & merge & & \\
\hline$O A$ & 92.7 & 79.5 & 81.2 & 80.3 & \begin{tabular}{|l|l|}
81.2 \\
\end{tabular} & 79.4 \\
\hline$A A$ & 92.1 & 79.7 & 80.3 & 80 & 80.4 & 79.6 \\
\hline$K$ & 0.85 & 0.59 & 0.61 & 0.6 & 0.61 & 0.59 \\
\hline C 1 & 87.9 & 68.8 & 74.1 & 70.6 & 73.8 & 68.7 \\
\hline C 2 & 96.3 & 90.6 & 86.6 & 89.3 & 86.9 & 90.6 \\
\hline & & esolt & ion 1 & nerge & & \\
\hline$O A$ & 92.7 & 84.1 & 91.3 & 91.3 & 90.9 & 84 \\
\hline$A A$ & 92.1 & 85.5 & 90.7 & 90.7 & 90.2 & 85.5 \\
\hline$K$ & 0.85 & 0.69 & 0.82 & 0.82 & 0.8 & 0.69 \\
\hline C 1 & & 71.4 & 89.9 & 86.9 & 85.7 & 71.4 \\
\hline $\begin{array}{ll}C 2 \\
\end{array}$ & 96.3 & 99.6 & 94.5 & 91.5 & 94.8 & 99.6 \\
\hline
\end{tabular}

\section{REFERENCES}

Aghighi, H., Trinder, J., Tarabalka, Y. and Lim, S., 2014. Dynamic block-based parameter estimation for MRF classification of high-resolution images. Geoscience and Remote Sensing Letters, IEEE 11(10), pp. 1687-1691.

Ardila, J. P., Tolpekin, V. A., Bijker, W. and Stein, A., 2011. Markov-random-field-based super-resolution mapping for identification of urban trees in VHR images. ISPRS Journal of Photogrammetry and Remote Sensing 66(6), pp. 762-775.

Atkinson, P., 1991. Optimal ground-based sampling for remote sensing investigations: estimating the regional meant. International Journal of Remote Sensing 12(3), pp. 559-567.

Atkinson, P. M., 1997. Mapping sub-pixel boundaries from remotely sensed images. Innovations in GIS 4, pp. 166-180.

Atkinson, P. M., Pardo-Iguzquiza, E. and Chica-Olmo, M., 2008. Downscaling cokriging for super-resolution mapping of continua in remotely sensed images. Geoscience and Remote Sensing, IEEE Transactions on 46(2), pp. 573-580.

Bagley, J. E., Desai, A. R., Harding, K. J., Snyder, P. K. and Foley, J. A., 2014. Drought and deforestation: Has land cover change influenced recent precipitation extremes in the Amazon. Journal of Climate 27(1), pp. 345-361.

Boucher, A. and Kyriakidis, P. C., 2006. Super-resolution land cover mapping with indicator geostatistics. Remote Sensing of Environment 104(3), pp. 264-282.

Boucher, A., Kyriakidis, P. C. and Cronkite-Ratcliff, C., 2008. Geostatistical solutions for super-resolution land cover mapping. Geoscience and Remote Sensing, IEEE Transactions on 46(1), pp. 272-283.
Bouman, C. A. and Shapiro, M., 1994. A multiscale random field model for bayesian image segmentation. Image Processing, IEEE Transactions on 3(2), pp. 162-177.

Clark, M. L., Roberts, D. A. and Clark, D. B., 2005. Hyperspectral discrimination of tropical rain forest tree species at leaf to crown scales. Remote Sensing of Environment 96(3), pp. 375398.

Erikson, M., 2003. Segmentation of individual tree crowns in colour aerial photographs using region growing supported by fuzzy rules. Canadian Journal of Forest Research 33(8), pp. 1557-1563.

Gong, P., Sheng, Y. and Biging, G., 2002. 3D model-based tree measurement from high-resolution aerial imagery. Photogrammetric Engineering \& remote sensing 68(11), pp. 1203-1212.

Halounov, L., 2003. Textural classification of B\& W aerial photos for the forest classification. In: EARSeL conference.

Houghton, R. A., 2005. Tropical deforestation as a source of greenhouse gas emissions. Tropical deforestation and climate change p. 13.

Houghton, R., Skole, D., Nobre, C. A., Hackler, J., Lawrence, K. and Chomentowski, W. H., 2000. Annual fluxes of carbon from deforestation and regrowth in the Brazilian Amazon. Nature 403(6767), pp. 301-304.

Jepma C.J., C. J., 2014. Tropical deforestation: a socio-economic approach. Taylor \& Francis.

Jing, L., Hu, B., Noland, T. and Li, J., 2012. An individual tree crown delineation method based on multi-scale segmentation of imagery. ISPRS Journal of Photogrammetry and Remote Sensing 70, pp. 88-98.

Kasetkasem, T., Arora, M. K. and Varshney, P. K., 2005. Super-resolution land cover mapping using a Markov random field based approach. Remote Sensing of Environment 96(34), pp. 302-314.

Koch, B., Heyder, U. and Weinacker, H., 2006. Detection of individual tree crowns in airborne lidar data. Photogrammetric Engineering \& Remote Sensing 72(4), pp. 357-363.

Li, X., Du, Y. and Ling, F., 2012. Spatially adaptive smoothing parameter selection for Markov random field based sub-pixel mapping of remotely sensed images. International Journal of Remote Sensing 33(24), pp. 7886-7901.

Li, X., Du, Y. and Ling, F., 2013. Super-resolution mapping of forests with bitemporal different spatial resolution images based on the spatial-temporal Markov random field. Selected Topics in Applied Earth Observations and Remote Sensing, IEEE Journal of PP(99), pp. 1-11.

Liguo, W., Qunming, W. and Danfeng, L., 2011. Sub-pixel mapping based on sub-pixel to sub-pixel spatial attraction model. In: Geoscience and Remote Sensing Symposium (IGARSS), 2011 IEEE International, pp. 593-596.

Ling, F., Li, X., Du, Y. and Xiao, F., 2013. Sub-pixel mapping of remotely sensed imagery with hybrid intra-and inter-pixel dependence. International Journal of Remote Sensing 34(1), pp. 341357.

Lu, D. and Weng, Q., 2005. Urban classification using full spectral information of Landsat ETM+ imagery in Marion county, Indiana. Photogrammetric Engineering \& remote sensing 71(11), pp. $1275-1284$. 
Lu, D. and Weng, Q., 2007. A survey of image classification methods and techniques for improving classification performance. International Journal of Remote Sensing 28(5), pp. 823870.

Luciani, P. and Chen, D., 2011. The impact of image and class structure upon sub-pixel mapping accuracy using the pixelswapping algorithm. Annals of GIS 17(1), pp. 31-42.

Makido, Y., Shortridge, A. and Messina, J. P., 2007. Assessing alternatives for modeling the spatial distribution of multiple landcover classes at sub-pixel scales. Photogrammetric Engineering and remote sensing 73(8), pp. 935.

Mas, J.-F., Puig, H., Palacio, J. L. and Sosa-Lpez, A., 2004. Modelling deforestation using GIS and artificial neural networks. Environmental Modelling \& Software 19(5), pp. 461-471.

Mertens, K. C., De Baets, B., Verbeke, L. P. and De Wulf, R. R., 2006. A sub-pixel mapping algorithm based on sub-pixel/pixel spatial attraction models. International Journal of Remote Sensing 27(15), pp. 3293-3310.

Mertens, K., Verbeke, L., Ducheyne, E. and De Wulf, R., 2003a. Using genetic algorithms in sub-pixel mapping. International Journal of Remote Sensing 24(21), pp. 4241-4247.

Mertens, K., Verbeke, L., Ducheyne, E. and De Wulf, R., 2003b. Using genetic algorithms in sub-pixel mapping. International Journal of Remote Sensing 24(21), pp. 4241-4247.

Powell, R. L., Roberts, D. A., Dennison, P. E. and Hess, L. L., 2007. Sub-pixel mapping of urban land cover using multiple endmember spectral mixture analysis: Manaus, Brazil. Remote Sensing of Environment 106(2), pp. 253-267.

Qunming, W., Wenzhong, S. and Liguo, W., 2014. Indicator cokriging-based subpixel land cover mapping with shifted images. Selected Topics in Applied Earth Observations and Remote Sensing, IEEE Journal of 7(1), pp. 327-339.

Richards, J. and Jia, X., 2006. Remote sensing digital image analysis an introduction. Springer, Germany.

Shen, Z., Qi, J. and Wang, K., 2009. Modification of pixelswapping algorithm with initialization fr om a sub-pixel/pixel spatial attraction model. Photogrammetric Engineering \& Remote Sensing 75(5), pp. 557-567.

Solberg, A. H. S., Taxt, T. and Jain, A. K., 1996. A markov random field model for classification of multisource satellite imagery. Geoscience and Remote Sensing, IEEE Transactions on 34(1), pp. 100-113.

Tatem, A. J., Lewis, H. G., Atkinson, P. M. and Nixon, M. S., 2001. Multiple-class land-cover mapping at the sub-pixel scale using a Hopfield neural network. International Journal of Applied Earth Observation and Geoinformation 3(2), pp. 184-190.

Tatem, A. J., Lewis, H. G., Atkinson, P. M. and Nixon, M. S., 2002. Super-resolution land cover pattern prediction using a Hopfield neural network. Remote Sensing of Environment 79(1), pp. 1-14.

Thornton, M., Atkinson, P. and Holland, D., 2006. Sub-pixel mapping of rural land cover objects from fine spatial resolution satellite sensor imagery using super-resolution pixel-swapping. International Journal of Remote Sensing 27(3), pp. 473-491.
Tolpekin, V. A. and Stein, A., 2009. Quantification of the effects of land-cover-class spectral separability on the accuracy of Markov-random-field-based superresolution mapping. Geoscience and Remote Sensing, IEEE Transactions on 47(9), pp. 3283-3297.

Tolpekin, V. A., Ardila, J. P. and Bijker, W., 2010. Superresolution mapping for extraction of urban tree crown objects from VHR satellite images. Small 3(1), pp. 1.

Verhoeye, J. and De Wulf, R., 2002. Land cover mapping at sub-pixel scales using linear optimization techniques. Remote Sensing of Environment 79(1), pp. 96-104.

Wang, Q., Wang, L. and Liu, D., 2012. Integration of spatial attractions between and within pixels for sub-pixel mapping. Systems Engineering and Electronics, Journal of 23(2), pp. 293-303.

Warner, T. A., Lee, J. Y. and McGraw, J. B., 1998. Delineation and identification of individual trees in the eastern deciduous forest. Automated interpretation of high spatial resolution digital imagery for forestry pp. 10-12.

Yong, X. and Bo, H., 2014. A spatio \& temporal pixel-swapping algorithm for subpixel land cover mapping. Geoscience and Remote Sensing Letters, IEEE 11(2), pp. 474-478.

Zahed, M. A., Ruhani, F. and Mohajeri, S., 2010. An overview of iranian mangrove ecosystem, northern part of the Persian gulf and Oman sea. Electronic Journal of Environmental, Agricultural and Food Chemistry 9(2), pp. 411-417.

Zhang, J., 2010. Multi-source remote sensing data fusion: status and trends. International Journal of Image and Data Fusion 1(1), pp. 5-24.

Zhang, L., Wu, K., Zhong, Y. and Li, P., 2008. A new sub-pixel mapping algorithm based on a BP neural network with an observation model. Neurocomputing 71(10), pp. 2046-2054.

Zhong, Y. and Zhang, L., 2013. Sub-pixel mapping based on artificial immune systems for remote sensing imagery. Pattern Recognition 46(11), pp. 2902-2926. 